\title{
A Multiscale Simulator for Low Pressure Chemical Vapor Deposition
}

\author{
Matthias K. Gobbert \\ Institute for Mathematics and its Applications, University of Minnesota, \\ 206 Church Street S.E. \#514, Minneapolis, MN 55455-0436, \\ gobbert@ima.umn.edu, phone: (612) 624-2838, fax: (612) 626-7370 \\ Tushar P. Merchant, Leonard J. Borucki \\ Advanced Custom Technologies, Motorola, Inc., Mail Drop M350, \\ 2200 W. Broadway Rd., Mesa, AZ 85202 \\ Timothy S. Cale \\ Center for Solid State Electronics Research, Arizona State University, \\ Tempe, AZ 85287-6206
}

\begin{abstract}
An integrated simulator for chemical vapor deposition is introduced. In addition to a reactor scale and feature scale simulators, it consists of a "mesoscopic" scale simulator with the typical length scale of a die. It is shown that the "three-scale" integrated simulator used is a proper extension of "two-scale" deposition simulators that consist of reactor scale and feature scale simulation models. Moreover, it is demonstrated that information is provided on a new length scale, for which no information is available from the "two-scale" approach, as well as important corrections to the simulation results on the reactor scale. This enables, for instance, studies of microloading. For these demonstrations, thermally induced deposition of silicon dioxide from tetraethyloxysilane (TEOS) is chosen as the application example, which is modeled by six gaseous reacting species involved in four gas-phase and eight surface reactions.
\end{abstract}

\section{Introduction}

The simulation of semiconductor manufacturing processes has become more important in recent years; many people consider it to be an integral tool in the development and evaluation of processes and equipment. The traditional simulation models include (1) reactor scale simulators, which solve the equations that govern the species and energy transport with chemical reactions throughout the reactor chamber, and (2) feature scale simulators, which solve the equations that govern the species transport and reaction inside one or more features, in order to predict the surface growth due to surface reactions. The length scale for reactor scale models is several tens of centimeters, while that for feature scale models is microns. These length scales may well diverge farther in the future, as reactors get larger and feature sizes decrease. Reactor scale models have been used to evaluate proposed reactor geometries in order to reduce the number of prototypes needed [1]. Feature scale models on the other hand have been used successfully to predict the evolution of film profiles and compositions 
inside features as functions of operating conditions, thus narrowing the operating window before test runs are performed [2].

In general, reactor scale and feature scale simulators have been used independently. Feature scale models require information regarding local species fluxes and temperature, which are generally inaccessible to measurement. One way to supply them is to use a reactor scale simulator to first predict the conditions throughout the reactor chamber based on macroscopic quantities like reactor set points $[3,4]$. The wafer is treated as flat by the reactor scale simulator. The predicted local conditions are then used in feature scale simulators at several positions of interest along the wafer surface. In this approach, a feature scale model could be used at each boundary node of the reactor scale model, which is assumed to lie inside a patterned region of the wafer.

This "two-scale" approach makes the tacit assumption that the reactor scale simulator with its mesh, that is coarse compared to the typical dimension of a feature scale simulator, is able to compute conditions above a particular feature entrance. Moreover, the structure of the wafer surface which can be represented by this two-scale approach is necessarily crude: When using a feature scale simulator at a particular boundary node of the reactor scale, the implicit assumption is made that this single feature is part of a larger cluster of identical features, for which it is a representative member. But in order to represent such a cluster appropriately in a numerical sense, several grid points across the cluster are needed, thus limiting the reactor scale simulator to modeling unrealistically large feature clusters. This approach has been used to obtain some information about deposition, particularly in the absence of loading [3,4]; however, the details of the surface structure of a realistic die with several much smaller clusters could not be represented there. Thus, feedback from the feature scale to the reactor scale, in order to account for the depletion of reactants in high pattern density regions of the wafer surface, is difficult with this approach.

A mesoscopic scale model and corresponding simulator have been introduced in order to model transport and reaction in a small region above the wafer surface on the scale of a few millimeters $[5,6,7,8,5,9]$. This scale deals with gradients on the length scale of dies ( $\mathrm{mm})$, and is intermediate with respect to the typical dimensions of reactor scale and feature scale models. The mesoscopic scale model is capable of resolving the surface structure inside a die consisting of several clusters of features. Notice that even in the coarsest implementation of this model, which assumes all features inside one cluster to be identical, there can be variations from one cluster to the others both in geometry and in reactor conditions. Indeed, an implementation using sufficiently many mesh points can even represent variations inside a cluster. The functionality of the mesoscopic scale model in stand-alone mode has been demonstrated in $[6,8]$.

As proposed in [9], the mesoscopic scale model has been designed to provide information on a length scale inaccessible to both reactor scale and feature scale models as well as to serve as an enhanced interface between those traditional models. This "three-scale" simulator couples numerical meshes whose typical mesh sizes are separated by fewer orders of magnitude than in the two-scale model. Moreover, due to the more meaningful feedback from the smaller scales to the larger ones, reactor scale simulations may account for the depletion or accumulation of chemicals close to the surface.

This work presents an implementation of the concept of a three-scale simulation model, in which a reactor scale, a mesoscopic scale, and several feature scale simulations are used in 


\begin{tabular}{|c|c||c|c|}
\hline Number & species & number & species \\
\hline 1 & $\mathrm{SiE}_{4}$ & 7 & vacancy \\
2 & $\mathrm{Si}(\mathrm{OH}) \mathrm{E}_{3}$ & 8 & $\mathrm{SiG} \mathrm{OH}) \mathrm{E}_{2}$ \\
3 & $\mathrm{O}\left(\mathrm{SiE}_{3}\right)_{2}$ & 9 & $\mathrm{SiGE}_{3}$ \\
4 & $\mathrm{H}_{2} \mathrm{O}$ & 10 & $\mathrm{SiG}_{3} \mathrm{E}$ \\
5 & $\mathrm{C}_{2} \mathrm{H}_{5} \mathrm{OH}$ & 11 & $\mathrm{SiG}_{3}(\mathrm{OH})$ \\
6 & $\mathrm{C}_{2} \mathrm{H}_{4}$ & 12 & $\mathrm{SiG}(\mathrm{OH})_{2} \mathrm{E}$ \\
\hline
\end{tabular}

Table 1: Nomenclature for the chemical species in the reaction chemistry.

unison to provide simulation information on all length scales of interest, and demonstrates that the approach taken is consistent with previous "two-scale" approaches; we "validate' our approach in this sense. Sections 2 and 3 explain the physical model and the simulation techniques, respectively. Section 4 presents the results obtained for the sample chemistry chosen, while Section 5 discusses the results and provides an outlook on future work.

\section{Model Description}

The chemical process chosen to demonstrate our three-scale simulation approach is thermally induced chemical vapor deposition in single wafer reactors. The following subsections detail the models and conditions used in this presentation.

Model Chemistry. - Thermally induced deposition of silicon dioxide $\left(\mathrm{SiO}_{2}\right)$ from tetraethyloxysilane (TEOS), with argon (Ar) as the carrier gas, is the example chosen for this demonstration. The kinetic model [10] involves six gaseous reacting species and one inert carrier gas, which participate in four gas-phase reactions and eight surface reactions. For the purposes of this paper, we focus attention on triethyloxysilane, a reactive intermediate formed from TEOS. It is a major contributor to film deposition in the chemistry model [10]. In the formulas below, the abbreviation $\mathrm{E}$ is used for the ethoxy group $\left(\mathrm{OC}_{2} \mathrm{H}_{5}\right)$. Moreover, $c_{i}$ denotes the gas-phase concentration of the $i$-th species in $\mathrm{mol} / \mathrm{cm}^{3}$, while $\nu_{i}$ stands for the (dimensionless) surface fraction of the $i$-th species. The species are counted according to the nomenclature in Table 1. Finally, $T$ denotes the temperature in $\mathrm{K}$, and the universal gas constant $R$ is equal to $1.987 \mathrm{cal} /(\mathrm{K} \mathrm{mol})$. The four gas-phase reactions used are

$$
\begin{aligned}
\mathrm{SiE}_{4} & \rightleftharpoons \mathrm{Si}(\mathrm{OH}) \mathrm{E}_{3}+\mathrm{C}_{2} \mathrm{H}_{4} \\
\mathrm{SiE}_{4}+\mathrm{H}_{2} \mathrm{O} & \rightleftharpoons \mathrm{Si}(\mathrm{OH}) \mathrm{E}_{3}+\mathrm{C}_{2} \mathrm{H}_{5} \mathrm{OH} \\
\mathrm{SiE}_{4}+\mathrm{Si}(\mathrm{OH}) \mathrm{E}_{3} & \rightleftharpoons \mathrm{O}\left(\mathrm{SiE}_{3}\right)_{2}+\mathrm{C}_{2} \mathrm{H}_{5} \mathrm{OH} \\
2 \mathrm{Si}(\mathrm{OH}) \mathrm{E}_{3} & \rightleftharpoons \mathrm{O}\left(\mathrm{SiE}_{3}\right)_{2}+\mathrm{H}_{2} \mathrm{O}
\end{aligned}
$$

with the reaction rates in $\mathrm{mol} /\left(\mathrm{s} \mathrm{cm}^{3}\right)$

$$
\begin{aligned}
& R_{1}=1.6 e^{-61450 / T} c_{1}-2.26 \cdot 10^{-3} e^{-48610 / T} c_{2} c_{6}, \\
& R_{2}=3.44 \cdot 10^{5} e^{-25000 / T} c_{1}-1.00 \cdot 10^{2} e^{-20970 / T} c_{2} c_{5}, \\
& R_{3}=2.78 \cdot 10^{4} e^{-30000 / T} c_{1} c_{2}-2.03 \cdot 10^{8} e^{-10900 / T} c_{3} c_{5}, \\
& R_{4}=2.78 \cdot 10^{4} e^{-30000 / T} c_{2}-7.01 \cdot 10^{11} e^{-14930 / T} c_{3} c_{4} .
\end{aligned}
$$


The eight surface reactions used are

$$
\begin{aligned}
& \mathrm{SiE}_{4}+\mathrm{SiG}_{3}(\mathrm{OH}) \rightleftharpoons \mathrm{SiO}_{2}(\mathrm{D})+\mathrm{SiGE}_{3}+\mathrm{C}_{2} \mathrm{H}_{5} \mathrm{OH}, \\
& \mathrm{SiGE}_{3} \rightleftharpoons \mathrm{SiG}_{3}(\mathrm{OH})+\mathrm{C}_{2} \mathrm{H}_{4} \text {, } \\
& \mathrm{SiG}(\mathrm{OH}) \mathrm{E}_{2} \rightleftharpoons \mathrm{SiG}(\mathrm{OH})_{2} \mathrm{E}+\mathrm{C}_{2} \mathrm{H}_{4} \text {, } \\
& \mathrm{SiGE}_{3} \rightleftharpoons \mathrm{SiG}(\mathrm{OH}) \mathrm{E}_{2}+\mathrm{C}_{2} \mathrm{H}_{4} \text {, } \\
& \mathrm{SiG}(\mathrm{OH})_{2} \mathrm{E} \rightleftharpoons \mathrm{SiG}_{3}(\mathrm{OH})+\mathrm{C}_{2} \mathrm{H}_{5} \mathrm{OH} \text {, } \\
& \mathrm{SiG}(\mathrm{OH}) \mathrm{E}_{2} \rightleftharpoons \mathrm{SiGE}_{3}+\mathrm{C}_{2} \mathrm{H}_{5} \mathrm{OH} \text {, } \\
& \mathrm{SiG}(\mathrm{OH})_{2} \mathrm{E} \rightleftharpoons \mathrm{SiG}_{3} \mathrm{E}+\mathrm{H}_{2} \mathrm{O} \\
& \mathrm{Si}(\mathrm{OH}) \mathrm{E}_{3}+\mathrm{SiG}_{3}(\mathrm{OH}) \rightleftharpoons \mathrm{SiO}_{2}(\mathrm{D})+\mathrm{H}_{2} \mathrm{O}+\mathrm{SiGE}_{3}
\end{aligned}
$$

with the reaction rates in $\mathrm{mol} /\left(\mathrm{s} \mathrm{cm}^{2}\right)$

$$
\begin{aligned}
& R_{1}=2.89 \cdot 10^{15} T^{0.5024} e^{-43820 / R T} c_{1} \nu_{11}-3.80 \cdot 10^{19} T^{-0.8715} e^{-47610 / R T} \nu_{v} \nu_{9} c_{5} \\
& R_{2}=1.7 \cdot 10^{12} e^{-46990 / R T} \nu_{9}-1.29 \cdot 10^{3} T^{2.001} e^{-34210 / R T} \nu_{11} c_{6}, \\
& R_{3}=3.4 \cdot 10^{12} e^{-61450 / R T} \nu_{8}-7.96 \cdot 10^{3} T^{1.915} e^{-34260 / R T} \nu_{12} c_{6} \\
& R_{4}=5.1 \cdot 10^{12} e^{-46990 / R T} \nu_{9}-2.24 \cdot 10^{4} T^{2.09} e^{-34510 / R T} \nu_{8} c_{6} \\
& R_{5}=2.01 \cdot 10^{12} e^{-44000 / R T} \nu_{12}-1.98 \cdot 10^{8} T^{0.5063} e^{-50620 / R T} \nu_{11} c_{5} \\
& R_{6}=2.00 \cdot 10^{12} e^{-44000 / R T} \nu_{8}-6.09 \cdot 10^{8} T^{0.4206} e^{-50670 / R T} \nu_{9} c_{5}, \\
& R_{7}=2.00 \cdot 10^{12} e^{-44000 / R T} \nu_{12}-1.26 \cdot 10^{12} T^{-0.3259} e^{-52170 / R T} \nu_{10} c_{4}, \\
& R_{8}=5.23 \cdot 10^{11} T^{0.8631} e^{-116680 / R T} c_{2} \nu_{11}-1.66 \cdot 10^{10} T^{2.410} e^{-13680 / R T} \nu_{v} c_{4} \nu_{9}
\end{aligned}
$$

Model of the Reactor. - The reactor is modeled as a generic single wafer reactor with stagnation point flow. Geometry as well as flow of the gases are assumed to be axi-symmetric. Therefore, the cross-section of one half of the reactor is sufficient to model the process. Figure 1 shows the geometry of the reactor chamber including some computed streamlines to indicate the flow profile throughout the reactor. Simulations are carried out for a $200 \mathrm{~mm}$ wafer on a $200 \mathrm{~mm}$ susceptor block housed in a $240 \mathrm{~mm}$ reactor chamber. The reactive gases enter at $300 \mathrm{~K}$ from the inlet situated $50 \mathrm{~mm}$ above the wafer. The wafer is assumed to be isothermally heated to $1000 \mathrm{~K}$. The reactor is modeled as a water-cooled cold wall reactor by imposing heat transfer conditions at the walls of the reactor chamber. The operating pressure of the reactor is $0.01 \mathrm{~atm}$, and the flowrate for the simulations is $2 \mathrm{slm}$.

Model of the Wafer Surface. - We consider the following three cases, all of which consider a flat $200 \mathrm{~cm}$ wafer with at most one die of non-flat area for demonstration purposes. The first case consists of a blanket wafer without any features. The second case considers a die between wafer radius $46.7 \mathrm{~mm}$ and $50.0 \mathrm{~mm}$. All features in the die are modeled as infinite trenches and taken as $1 \mu \mathrm{m}$ in depth and $1 \mu \mathrm{m}$ in width, hence with aspect ratio 1 , and with flat areas of $2 \mu \mathrm{m}$ separating the features, resulting in a pitch of $3 \mu \mathrm{m}$. These two cases are used to validate the three-scale approach, since they agree with geometries that a two-scale simulator is capable of representing. The third surface case attempts to pose a more realistic geometry, in which the die does not consist of uniform features but rather contains three clusters of features of width $0.4 \mathrm{~mm}$ each. The features are of the same kind as those in the second case. Figure 1 shows the position of the $3.3 \mathrm{~mm}$ wide die as well as the arrangement of clusters within the die. 


\section{Simulation Method}

For the purposes of the presentation here, we restrict our attention to obtaining the pseudo steady-state solution for the deposition model, which might then be used as the initial solution for a transient deposition simulation. Due to the feedback from the other models, each model's solution must satisfy the governing equations on its own scale and be consistent with the ones obtained on the other scales, and hence an iterative solution is necessary. The following three subsections provide details of the models used on the three length scales, while the fourth one discusses the crucial issues relating to the interfaces between them both for a two-scale deposition model as well as for the three-scale integrated deposition model.

Reactor Scale Model. - The reactor scale is modeled by the equations from classical continuum mechanics. Specifically, the two-dimensional axi-symmetric Navier-Stokes equations, the continuity equation, the energy equation, and six species balance equations are solved [11]. The simulations on the reactor scale are performed using the software package FIDAP 7.6 [12], which is a fluid dynamics simulation tool based on finite elements. The velocity, temperature, and mass fractions are interpolated using biquadratic basis functions, whereas the pressure is interpolated bilinearly. Figures 2 and 3 show the setup of the simulators for the two-scale and for the three-scale model, respectively. Both show the numerical mesh of the left half of the reactor chamber, the domain of the reactor scale model. Not shown is the (uniform) mesh used for the susceptor block. Figure 3 also shows the mesh for the mesoscopic scale model, while both figures include arrows which indicate the position of the feature scale models.

Mesoscopic Scale Model. - The mesoscopic scale model is designed to resolve the region of the gas-phase over a die-sized part of the wafer. As stated above, the die is assumed to be $3.3 \mathrm{~mm}$ wide, and the domain is chosen to extend $1 \mathrm{~mm}$ into the gas-phase. The velocity and the total pressure are assumed to be known throughout this region. With a typical length scale of $1 \mathrm{~mm}$ and a mean free path of less than $0.3 \mathrm{~mm}$ for all species, the Knudsen number is at most 0.3 in the mesoscopic scale model. Hence, it is reasonable to approximate the solution by the equations of continuum flow. Therefore, six diffusion-reaction equations and the energy equation are solved for the mass fractions of the six gaseous species and the temperature. The simulation package FIDAP 7.6 [12] is used to compute the solution on the mesoscopic scale. A uniform grid of size 16-by-16 is used for the domain of size $3.3 \mathrm{~mm}$ by $1 \mathrm{~mm}$, as shown in Figure 3.

Feature Scale Model.-On the feature scale, the species transport inside a single feature with typical length scale of at most $1 \mu \mathrm{m}$ must be described as molecular flow. Therefore, it is modeled by the Ballistic Transport and Reaction Model [2] implemented in the software package EVOLVE 4.1a [13]. The larger scale model passes the species concentrations, the temperature, and the total pressure at the feature entrance to EVOLVE, from which it computes the total flux into the surface of the feature. The arrows in Figures 2 and 3 point to the boundary nodes at which the transport and reaction in a representative feature is simulated by EVOLVE. Since only a pseudo steady-state is computed at present, the surface is not moved. 
Combining the Models. - A two-scale deposition simulator is obtained by directly combining the reactor scale simulator with the feature scale simulator applied to representative features at several positions of the wafer. Figure 2 shows the arrangement used to study a single patterned die on an otherwise flat wafer. The feature scale simulator is used at each of the three boundary nodes of the sole finite element covering the position of the patterned die. Practically, it is called by the reactor scale simulator at every iteration in order to determine the total flux into the surface at the boundary node based on the current iterate's value for the species concentrations there.

The three-scale deposition model is based on the premise that such a two-scale combination is problematic, because the length scales which have to be bridged are separated by five orders of magnitude. The mesoscopic scale model is introduced to improve the accuracy of the information interchange between the reactor scale and the feature scale models as well as the validity of the reactor scale model by taking into account the feedback of information from the smaller scales. By construction, the mesoscopic scale is chosen to agree with the size of the patterned die to be modeled. On this scale, it becomes possible to resolve the individual clusters of features contained in the die, as indicated by the three groups of arrows in Figure 3, each of which points at a boundary node of the mesoscopic scale model that lies inside a clustered region of the die. The mesoscopic scale model is used to supply the species fluxes into the boundary based on the current conditions at the three nodes of the boundary element of the reactor scale grid.

More precisely, the input from the reactor scale model to the mesoscopic scale model consists of the current iterates' values at the left side, the center, and the right side of the mesoscopic scale domain. These values are interpolated by a quadratic function, whose values are used as input to the feature scale models. In turn, at each boundary node of the mesoscopic scale model, the feature scale model returns the value of the net flux into the surface, which are used to prescribe the boundary condition of the mesoscopic scale model at its boundary nodes. A new iterate of the mesoscopic scale model is then computed. If the new solution of the mesoscopic scale model still differs from the previous one, the feature scale models are called again to prescribe updated boundary condition values, and a new iterate of the mesoscopic scale model is computed. Once the mesoscopic scale model is converged, the values of the net flux into the surface at the left side, the center, and the right side of the mesoscopic domain have to be returned to the reactor scale model to prescribe its boundary conditions at its three nodes of the element. These three values are obtained from a linear function, whose two coefficients are determined by the following two conditions: (1) The total flux through the boundary of the reactor scale has to agree with the one through the mesoscopic scale, and (2) the linear function approximates the flux values from the feature scale model in a least squares sense. Finally, the reactor scale model has its boundary conditions prescribed and computes a new iterate itself. The procedure is then repeated, until the solution on the reactor scale is converged.

In the approach outlined above for two-scale modeling, a fully patterned and a partially patterned die are indistinguishable both to the reactor scale and the feature scale model. This is still true for these two models as individual parts of the three-scale model, but their converged solutions are consistent with the solution of the mesoscopic scale model, which is also converged for the resolved internal die structure, and hence the overall three-scale model accounts for the pattern structure inside the die. 
It should be pointed out that this presentation uses the three-scale deposition model with only one mesoscopic scale model at the sole patterned die on the wafer. This geometry has been chosen to focus the comparisons on one area of the wafer. In practice, the three-scale deposition model would use (at least) one mesoscopic scale model for each patterned die on the wafer.

\section{Results}

The first two cases considered are designed to 'validate' the implementation of the threescale deposition model. To this end, the three-scale model consisting of the reactor scale, the mesoscopic scale, and the feature scale models is tested against the two-scale implementation using only the reactor scale and the feature scale models in direct interaction. The cases are first chosen for the two-scale model, then the appropriate equivalent cases are simulated with the three-scale model. This procedure is used to ensure that we compare results for cases which the two-scale model is capable of representing. It is also a consequence of the last criterion that all comparisons have to be taken on the reactor scale. For the purpose of this presentation, triethyloxysilane (a reaction intermediate formed in the gas-phase) is used as the representative gaseous species, and all graphs refer to it. However, equivalent results have been obtained for all other gaseous species.

The first case checks whether both models yield the same result for a geometry using a blanket wafer without any features. Figure 4 shows the mass fraction of triethyloxysilane along the wafer surface vs. the wafer radius. The solid curve is obtained from the two-scale model, while the circled data result from the three-scale model. Very good agreement is observed. To analyze the difference more explicitly, Figure 5 shows a plot of the difference between the mass fraction in the two solutions in Figure 4. The maximum difference in mass fraction is seen to be less than $4.0 \cdot 10^{-9}$ or $0.003 \%$. This basic validation shows that the three-scale model solves the case of a blanket wafer correctly, since it yields the same result on the reactor scale as the two-scale model, which is also capable of representing this surface case.

The second validation case assumes a geometry in which one die is located approximately at the center of the reactor scale domain, as described above. Notice that this die agrees precisely with the size of one boundary element of the finite element mesh. Since quadrilateral basis functions are used, three nodes are located along this boundary element. For the twoscale model, the feature scale model is used at these three nodes to compute the species fluxes due to surface reactions. For the three-scale model to treat an equivalent case, this geometry implies that all boundary nodes of the mesoscopic scale model lie in one patterned area, and the feature scale model is used at all mesoscopic boundary nodes.

Figure 6 shows the mass fraction of triethyloxysilane vs. the wafer radius obtained from both models. The agreement is very good, as is also demonstrated by the plot of the difference shown in Figure 7 . The maximum difference in mass fraction is less than $4.0 \cdot 10^{-8}$ or $0.035 \%$. This case demonstrates again that the three-scale deposition model yields the same result on the reactor scale as the two-scale model for the case that both 'see' the same surface case.

As the third case, we choose the more realistic case of a die containing three clusters of features, as described in detail above. For this case, notice that the reactor scale mesh still 
has only three boundary nodes available to resolve the surface area of the die. As a result, the two-scale model produces exactly the same result as for the second surface case above, since it does not account for the flat areas which occur inside the die. On the other hand, the mesoscopic scale model is designed to account for the flat areas, while it considers each cluster of features to consist of the representative features at the surface nodes inside each cluster.

Figures 8 and 9 show contour plots of the mass fraction of triethyloxysilane throughout the reactor chamber and in the mesoscopic scale domain, respectively. In Figure 8, the contour lines range from 0 to $1.3 \cdot 10^{-4}$ with the maximum attained at the center of the wafer. This is expected since the species is an intermediate, which is generated by gasphase reactions, and which is consumed by the surface reactions. This is evident in the mesoscopic scale result in Figure 9, which provides a close-up picture of the mass fraction of triethyloxysilane close to the wafer surface: The contour lines range from $1.12 \cdot 10^{-4}$ to $1.16 \cdot 10^{-4}$ with the maximum attained at the right side of the domain just above the center. That means that there is net generation of the species in the gas-phase of the mesoscopic scale domain, while very close to the surface depletion dominates.

Figure 10 contains plots of the mass fraction of triethyloxysilane along the wafer surface vs. the wafer radius. The three curves are obtained by using the three-scale deposition model for each of these cases. As discussed above, the curves of the two-scale model for the first and second surface case agree with the corresponding ones of the three-scale model. Notice first that all three curves agree throughout the flat regions of the wafer. Secondly, since triethyloxysilane is depleted due to surface reactions, the mass fraction decreases with increasing total surface area available for deposition. Hence, in the area inside the die, the curve for the third case lies in between the curves for the other cases. This is appropriate, since as more surface area is available for deposition, the species is depleted more in the surface reactions.

Finally, Figure 11 shows the same quantities as Figure 10, but now on the length scale of the mesoscopic scale model. As expected, the level of the mass fraction decreases again with an increase in total surface area, hence the curve for the second case is lower than the one for the first case. The curves for the flat wafer and the uniformly patterned die do not exhibit any significant variations, which is correct for their uniform surface model on the mesoscopic scale. Notice that the variations visible for the surface involving three clusters in the die can only be captured by the mesoscopic scale model. It provides information in this case, for instance on microloading, which is not accessible to the other models, since these cannot resolve the details of the surface structure inside the die.

Finally, Figure 12 shows the film profile of the deposited silicon dioxide, if deposition is continued for 20 minutes at the modeled deposition rate. Since only a pseudo steady-state solution is computed here, this results only demonstrates the conformality of the deposition process. With the three-scale simulator used in transient mode, a deposition history could be obtained on the feature scale in this way.

\section{Conclusions and Discussion}

This presentation has demonstrated that the three-scale deposition model agrees with the two-scale model for a flat wafer as well as for a uniformly patterned die. This shows the 
three-scale model to be a consistent extension of the two-scale model, since it preserves its predictions for the cases which the two-scale model is capable of resolving.

However, the three-scale deposition model provides information on a new length scale, namely on the scale of feature clusters inside a die. For instance, the information about the phenomenon of microloading as seen in Figure 11 for the case of three clusters of features inside the die cannot be obtained from the two-scale model. This fact has already been demonstrated for the mesoscopic scale model in stand-alone mode and using a single-species approximation to the same chemistry in [8]. These results highlight at least two aspects of simulations; (1) the model equations have to be valid for the size of the domain, and (2) the numerical method used must be able to sufficiently resolve the scale of interest.

As implemented, the mesoscopic scale model converges to its solution very quickly, usually in less than five iterations, independent of the initial guess used. Hence, the mesoscopic scale model appears to be quite a robust tool, which is an important property for a larger scale implementation using a number of intermediate scale models. Notice that such an arrangement could involve mesoscopic scale models at several positions of the wafer as well as several levels of such models forming a hierarchy of models on every length scale of interest to the user.

Despite the robustness of the mesoscopic scale model, there is need for improved speed of the overall simulations. This is mainly related to the time required for the setup of FIDAP (as well as EVOLVE) simulations. As one step towards this end, the current implementation already calls the mesoscopic scale model only every ten iterations of the reactor scale model. This number may need to be fine-tuned for each different chemistry under consideration, depending, among other things, on how much the reactor scale solution changes from one iteration to the next one.

A reasonable direction for development of a three-scale deposition model which involves several mesoscopic scale and several feature scale domains is to parallelize these simulators, since they are only coupled through the reactor scale. This approach should also be used for each individual mesoscopic scale model as well as for the reactor scale model's flat areas of the wafer. In both cases, the feature scale model is called numerous times without any interdependence.

Even more immediate savings could be achieved if mesoscopic scale simulations could be avoided altogether. This idea is based on the observation that the only quantity returned from the feature scale model is the species flux into the surface at one feature, the size of which largely depends on the effective surface area of that feature [5, 7]. The same might hold for the flux values returned from the mesoscopic scale to the reactor scale model, if one considers Figure 10. Future work is needed to determine the validity of the idea for combinations of chemistries and surface structure. Notice that in any case, the information provided by the mesoscopic scale model about the effects on the die scale would be lost. Moreover, the advantages with respect to efficiency of the simulations have to be weighed against the loss of accuracy of the interface, in particular considering the increasing difference in length scales of reactor scale (larger wafers) and feature scale models (higher integration).

Another area of future research concerns the pressure regime of the reactor. While we believe that the continuum assumption provides a model which approximates the physics quite well for the regime used here, an extension to lower pressures (higher Knudsen numbers) is needed to accommodate several important examples of operating conditions. 


\section{Acknowledgments}

This work was partially supported by the Semiconductor Research Corporation, the National Science Foundation, the Defense Advanced Research Projects Agency, and Motorola, Inc.

\section{REFERENCES}

1. D. W. Studiner, J. T. Hillman, R. Arora, and R. F. Foster, in Advanced Metallization for ULSI Applications 1992, T. S. Cale and F. Pintchovski, Editors, p. 211, Materials Research Corporation (1993).

2. T. S. Cale and V. Mahadev, in Modeling of Film Deposition for Microelectronic Applications, S. Rossnagel and A. Ulman, Editors, vol. 22 of Thin Films, p. 175, Academic Press (1996).

3. A. Hasper, J. Holleman, J. Middelhoek, C. R. Kleijn, and C. J. Hoogendoorn, J. Electrochem. Soc., 138, 1728 (1991).

4. T. S. Cale, J.-H. Park, T. H. Gandy, G. B. Raupp, and M. K. Jain, Chem. Eng. Commun., 119, 197 (1993).

5. M. K. Gobbert and C. A. Ringhofer, SIAM J. Appl. Math., Accepted.

6. M. K. Gobbert, T. S. Cale, and C. A. Ringhofer, in Process Control, Diagnostics, and Modeling in Semiconductor Manufacturing, M. Meyyappan, D. J. Economou, and S. W. Butler, Editors, PV 95-2, p. 553, The Electrochemical Society Proceedings Series, Pennington, NJ (1995).

7. M. K. Gobbert, T. S. Cale, and C. A. Ringhofer, Proceedings of the Fourth International Workshop on Computational Electronics, Tempe, AZ, Nov. 1995.

8. M. K. Gobbert, C. A. Ringhofer, and T. S. Cale, J. Electrochem. Soc., 143, 2624 (1996).

9. M. K. Gobbert, T. P. Merchant, T. S. Cale, and L. J. Borucki, in Advanced Metalization and Interconnect Systems for ULSI Applications in 1996, R. Havemann and J. Schmitz, Editors, MRS Proceedings Series, In press.

10. P. Ho (Sandia National Laboratories), presented at the Schumacher Forum for Future Trends, San Diego, CA, Feb. 1995, and private communication.

11. R. B. Bird, W. E. Stewart, and E. N. Lightfoot, Transport Phenomena. John Wiley \& Sons, Inc., New York (1960).

12. FIDAP 7.6, Fluid Dynamics International, 500 Davis St., Ste. 600, Evanston, IL 60201 (1996).

13. EVOLVE is a low pressure transport and reaction simulator developed by Timothy S. Cale at Arizona State University and Motorola, Inc. with funding from the Semiconductor Research Corporation, the National Science Foundation, and Motorola. EVOLVE 4.1a was released in February 1996. 


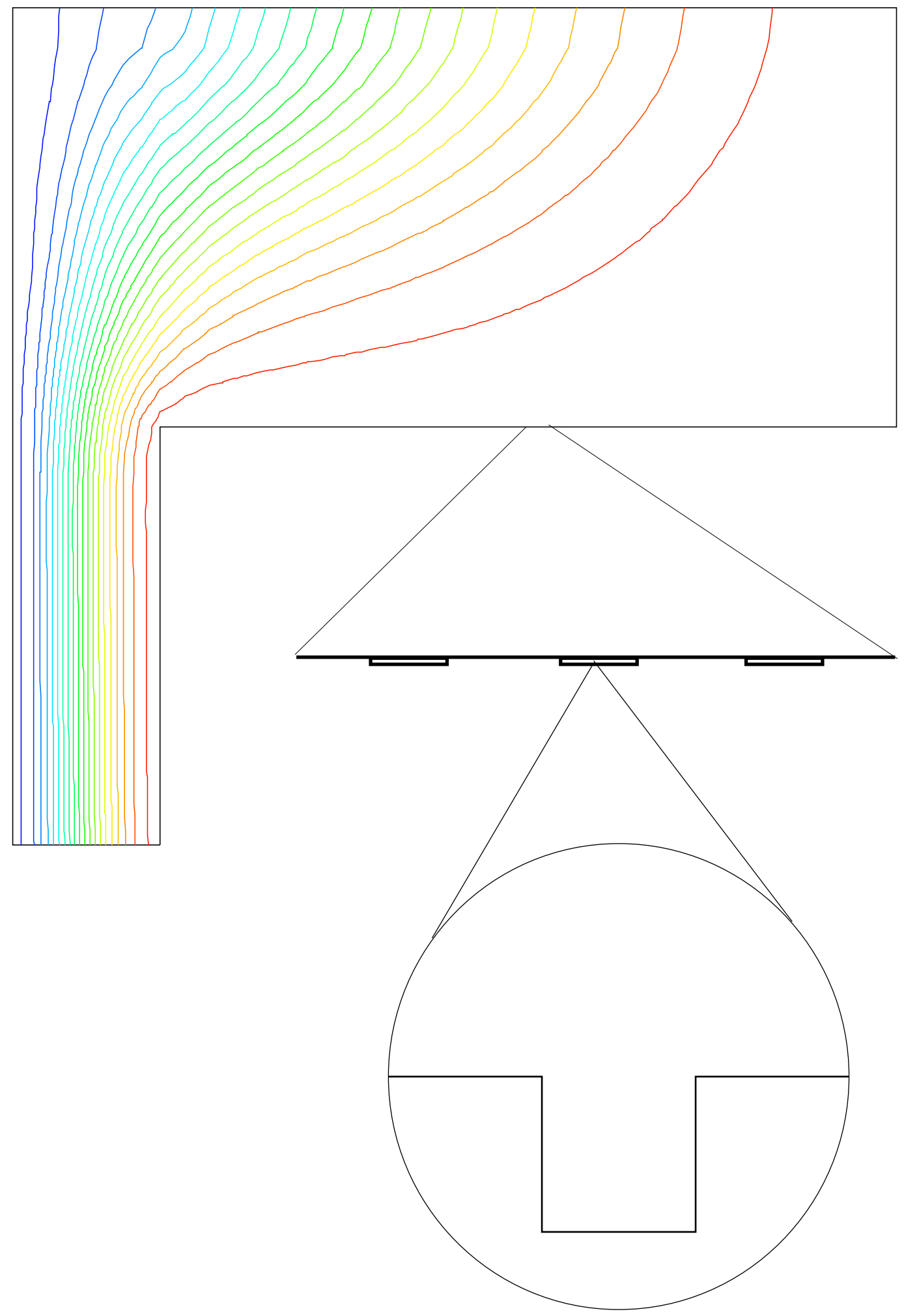

Figure 1: Sketch of the geometry for the three-scale deposition model. 


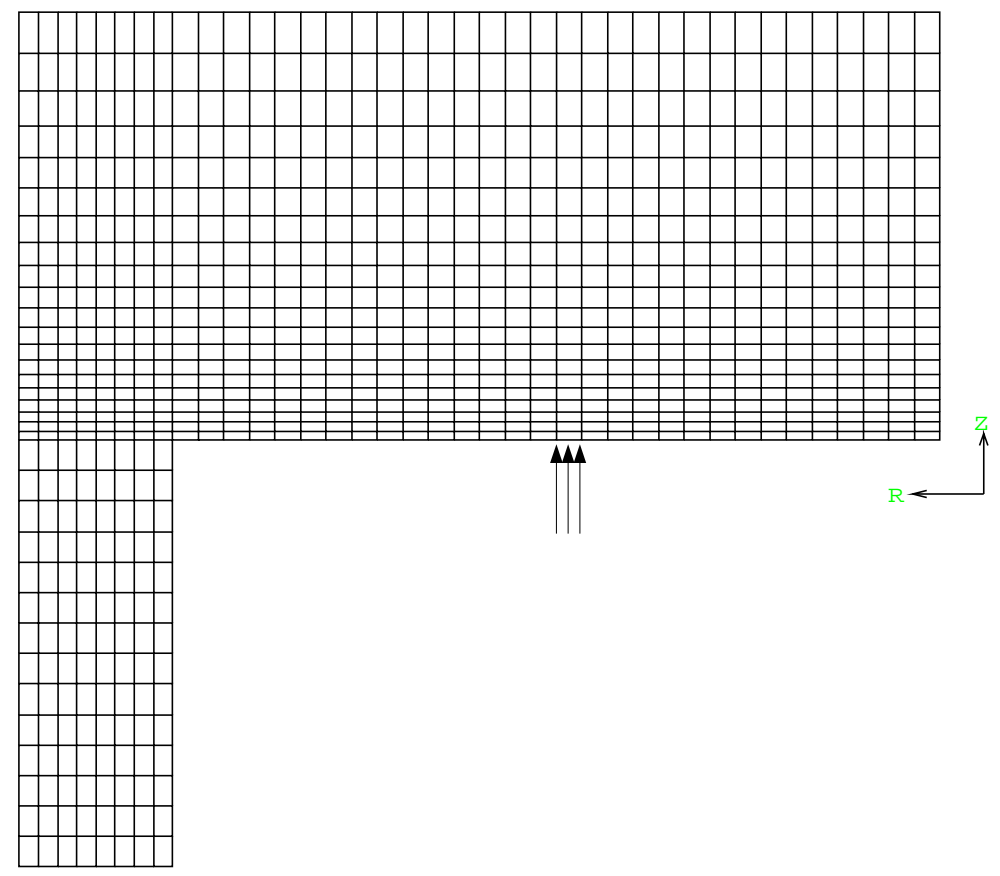

Figure 2: Schematic of the setup for a two-scale deposition model and the mesh of the reactor scale model. The arrows indicate the positions of the feature scale models.

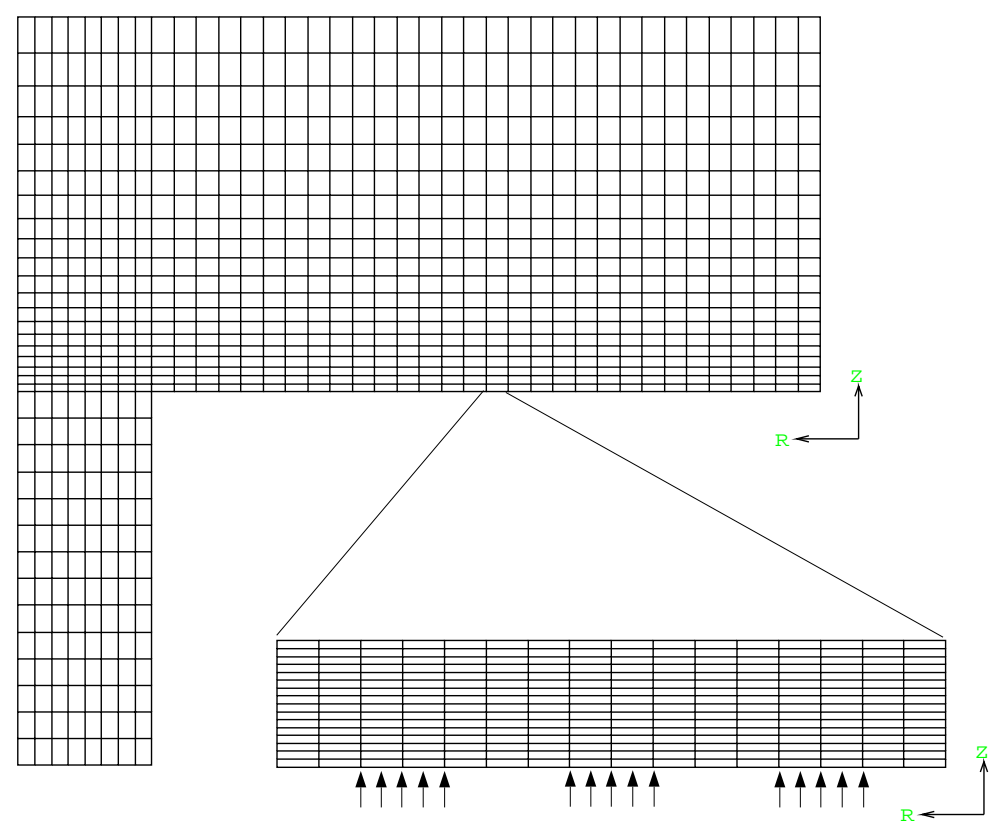

Figure 3: Schematic of the setup for the three-scale deposition model and the meshes of the reactor scale and mesoscopic scale models. The arrows indicate the positions of the feature scale models. 


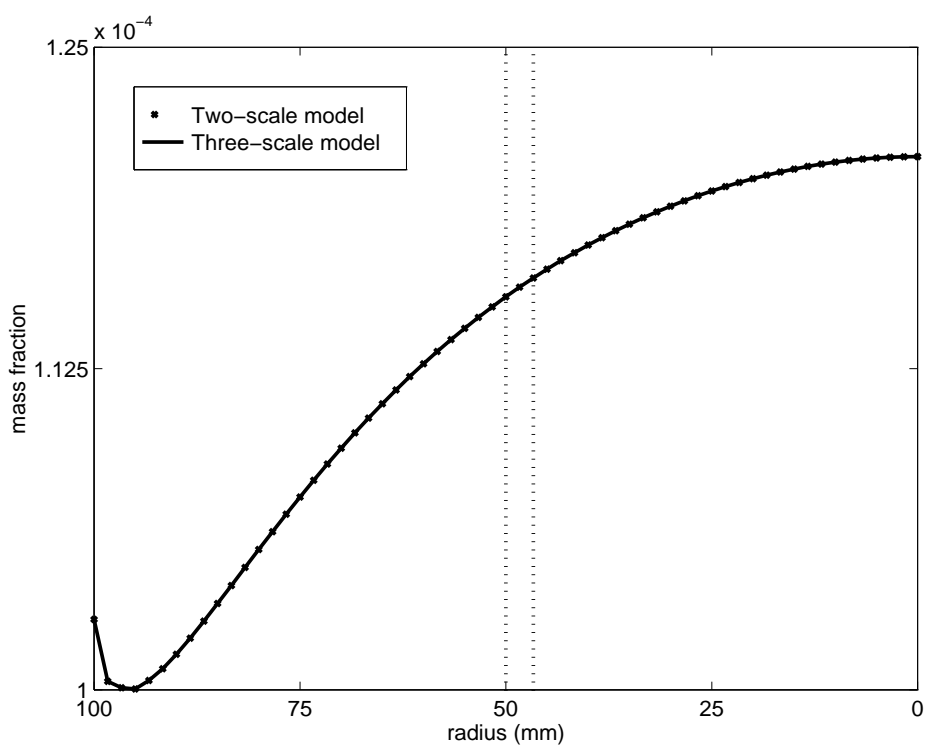

Figure 4: Mass fraction of triethyloxysilane vs. wafer radius for a blanket wafer. The dotted lines mark the location of the die on the wafer surface.

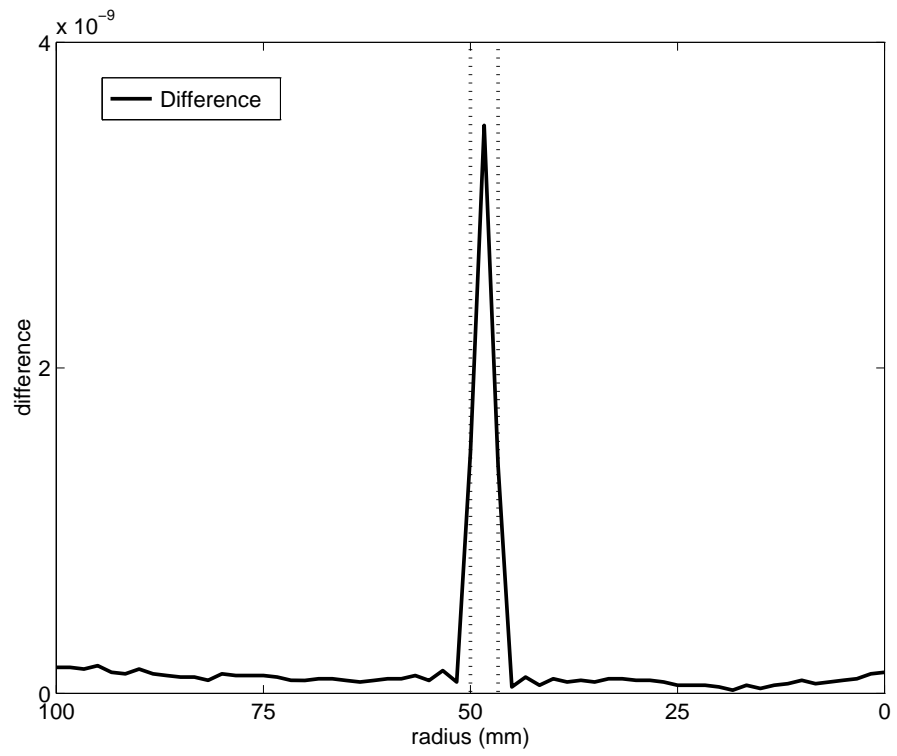

Figure 5: Difference of mass fraction of triethyloxysilane vs. wafer radius for a blanket wafer. The maximum relative difference is less than $0.003 \%$. The dotted lines mark the location of the die on the wafer surface. 


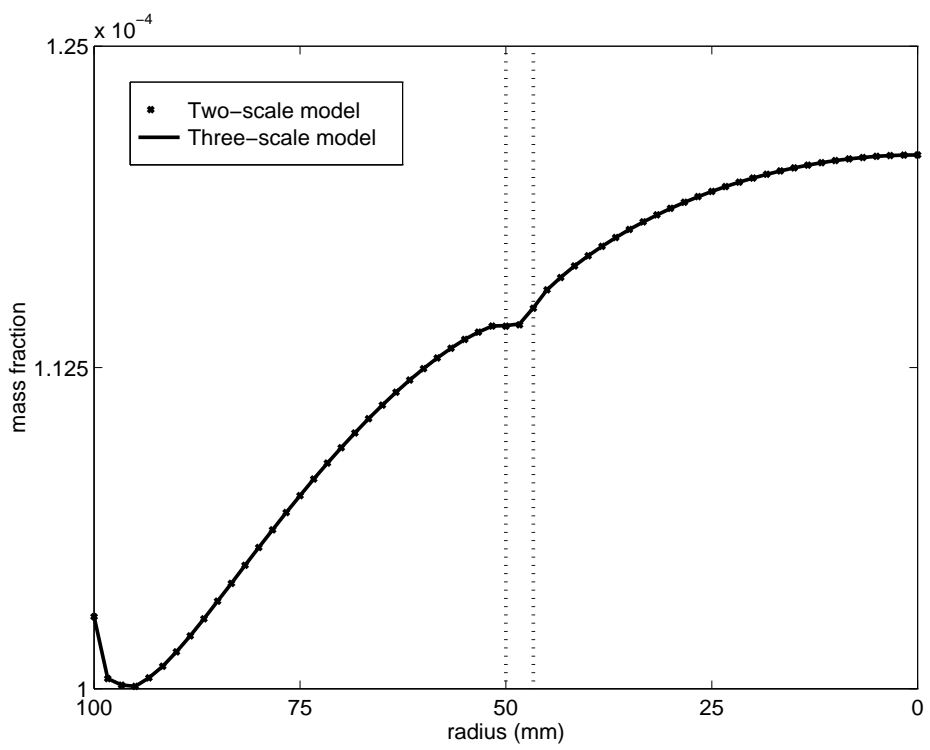

Figure 6: Mass fraction of triethyloxysilane vs. wafer radius for a wafer with one uniformly patterned die. The dotted lines mark the location of the die on the wafer surface.

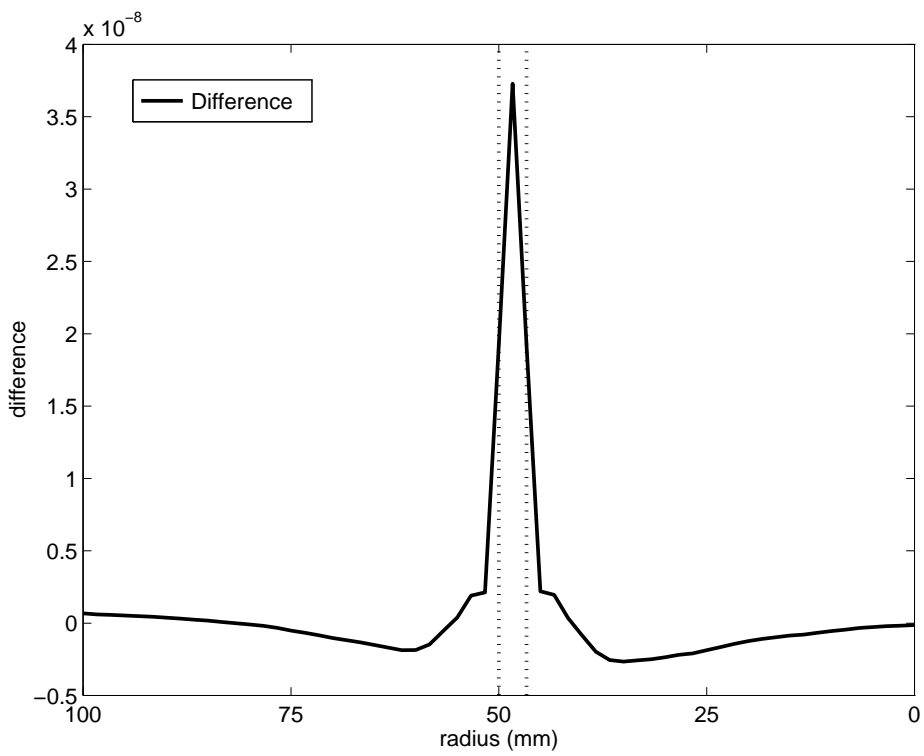

Figure 7: Difference of mass fraction of triethyloxysilane vs. wafer radius for a wafer with one uniformly patterned die. The maximum relative difference is less than $0.035 \%$. The dotted lines mark the location of the die on the wafer surface. 


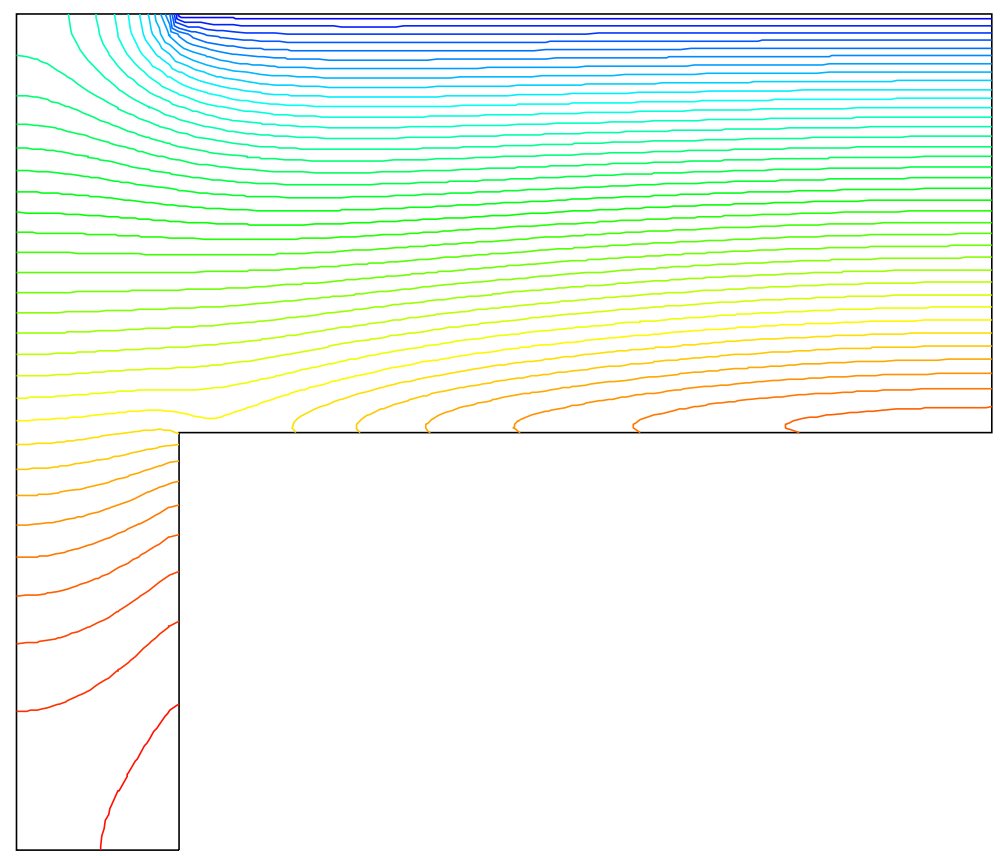

Figure 8: Contour plot of the mass fraction of triethyloxysilane throughout the reactor chamber. The contour lines range from 0 to $1.3 \cdot 10^{-4}$ with the maximum attained at the center of the wafer in the lower right corner of the plot.

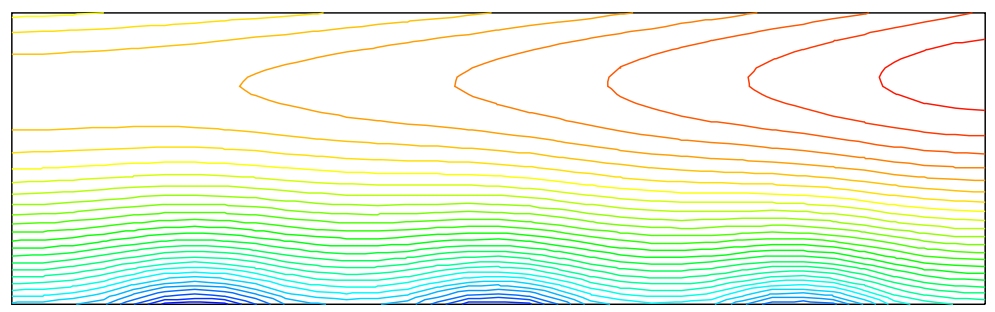

Figure 9: Contour plot of the mass fraction of triethyloxysilane throughout the mesoscopic scale domain. The contour lines range from $1.12 \cdot 10^{-4}$ to $1.16 \cdot 10^{-4}$ with the maximum attained at the crest on the right end of the domain. 


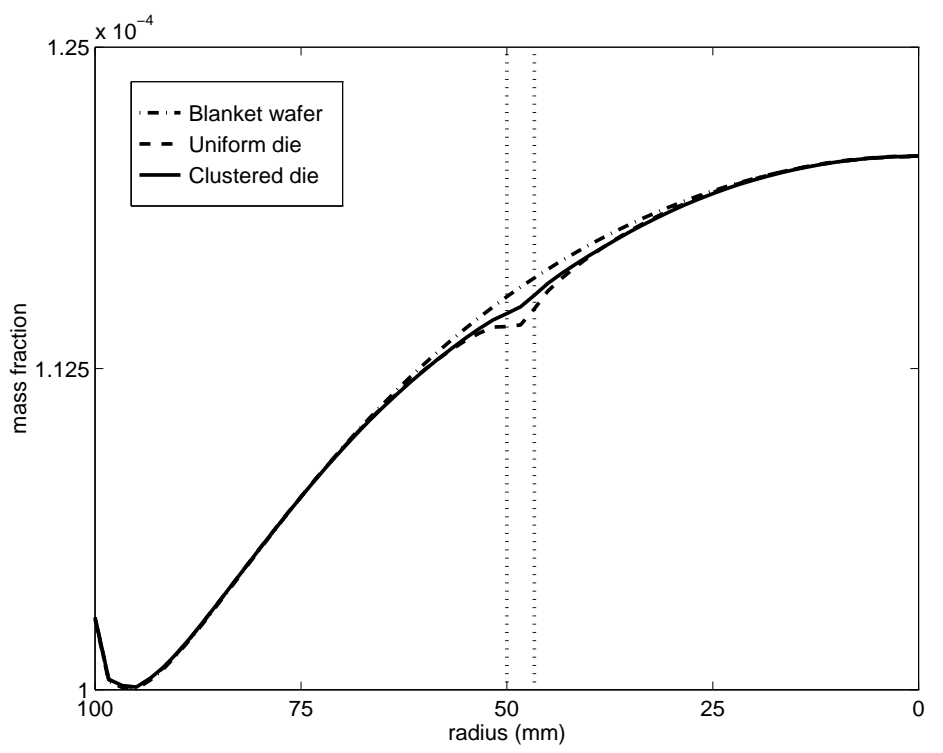

Figure 10: Mass fraction of triethyloxysilane vs. wafer radius across the wafer. The dotted lines mark the location of the die on the wafer surface.

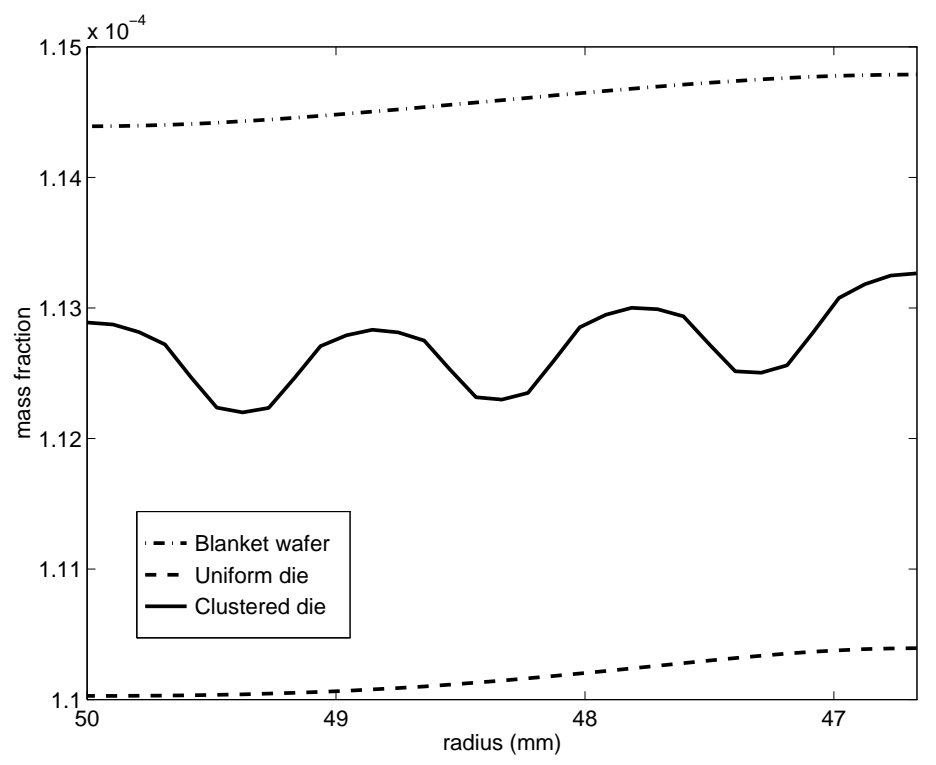

Figure 11: Mass fraction of triethyloxysilane vs. wafer radius across the die. The dotted lines mark the location of the die on the wafer surface. 


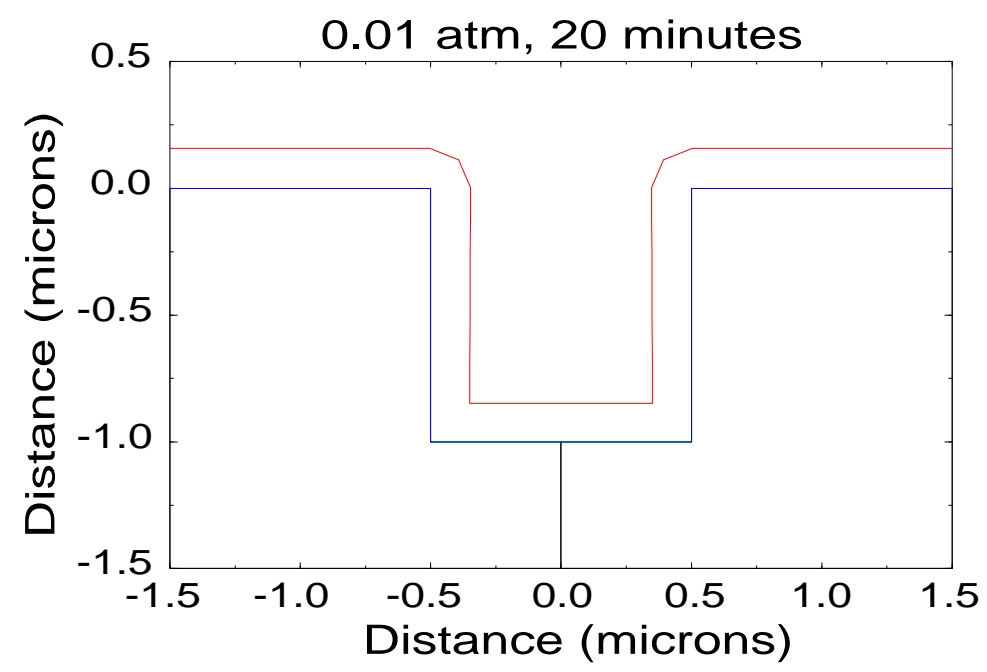

Figure 12: Film profile of deposited silicon dioxide after 20 minutes. 